\title{
Skeletal Class III Treated with Double Jaw Surgery: A Case Report
}

\section{Chaima Attia ${ }^{1 *}$, Ines Dallel ${ }^{2}$, Ramzi Moatemri ${ }^{3}$, Samir Tobji ${ }^{2}$ and Adel Ben Amor ${ }^{2}$}

${ }^{1}$ Doctors (DDS), University of Monastir, Faculty of Dental Medicine, Tunisia

${ }^{2}$ Professors, PHD - University of Monastir, Faculty of Dental Medicine, Dento-Facial

Orthopedics Department of Monastir Dental Clinic, Laboratory of Oral Health and

Orofacial Rehabilitation, Tunisia

${ }^{3}$ Professors, PHD - University of Sousse, Faculty of Medicine of IBN EL JAZZAR of

Sousse, Maxillofacial surgery Department of the university hospital of Sahloul,

Tunisia

*Corresponding Author: Chaima Attia, Doctors (DDS), University of Monastir, Faculty of Dental Medicine, Tunisia.
Received: December 09, 2021

Published: January 06, 2022

(C) All rights are reserved by Chaima Attia., et al.

\begin{abstract}
A female patient with severe class III skeletal malocclusion and hypodivergent face was treated with a conventional orthognatic treatment. Our patient had a dental class III canine and molar relationship, an anterior cross bite and a gummy smile. Her severe anomaly was responsible of some functional issues such as TMJ disorder and difficulties at mastication. We observed a straight profile with a shy and narrow smile. The treatment began with orthodontic surgery preparation(decompensation) that lasted 24 months. Then a bimaxillary surgery was conducted with Lefort 1 osteotomy of advancement and impaction, associated to mandibular osteotomy in order to rotate the lower jaw. Finally, the finishing orthodontic phase had an objective: to close posterior open bite with intermaxillary elastics. Important amelioration of esthetics, oral functions and especially better self-esteem of the patient were obtained. Cephalometric superposition demonstrated that pharyngeal airways width was improved and skeletal discrepancy was corrected.
\end{abstract}

Keywords: Skeletal Class III; Orthognatic Treatment; Pharyngeal Airway; Bimaxillary Surgery

\section{Introduction}

Class III skeletal malocclusion is one of the most difficult occlusal anomalies to be treated. In front of such cases, four procedures are available to achieve esthetic and functional goals: an orthopedic early treatment trying to modify growth so that jaw discrepancy is reduced or resolved; a compensatory orthodontic treatment or an orthodontic "camouflage"; the combination of those two options and finally a surgical orthognathic treatment to get a reposition of the skeletal bases $[1,2]$.

- $\quad$ The class III skeletal discrepancy may results from an excessive mandibular growth (mandibular prognathism); a maxil- lary retrusion or deficiency (maxillary brachygnathia); or the combination of both [3]. Primarily, the diagnostic of skeletal class III is based on imaging, ANB angle and AoBo measurement. Some authors have used CBCT [4]. It was improved that $3 \mathrm{D}$ image may be highly reproducible regardless to $2 \mathrm{D}$ radiographs.

- $\quad$ According to a recent study in Tunisian population, class III malocclusion represents $9,3 \%$ of the total cases treated [5].

- $\quad$ As we have already mentioned, early treatment followed by a "camouflage" orthodontic treatment is the best way to treat a class III resulting from a maxillary deficiency [5]. Usually, this 
orthopedic treatment is associated to a rapid maxillary expansion (RME) by opening the mid palatal suture [6].

- Knowing that is patent in young children; this protocol of RME stimulates all the suture system of the maxilla to facilitate the $\mathrm{A}^{\circ} \mathrm{P}$ action of the facial mask [7]. However, after the growth period, a maxillary Le fort I osteotomy and an orthodontic surgical treatment is indicated.

- $\quad$ This case report presents the treatment of a class III skeletal malocclusion with a sagittal and a transverse deficiency: Maxillary brachygnatia with a severity that required an orthodontic and surgical treatment to reach a normal and functional occlusion and a facial esthetics.

\section{Dental clinical examination and diagnosis}

- $\quad \mathrm{RB}$, a 24-year-old Tunisian female, presented to the department of orthodontics at the dental medicine clinic of Monastir. Her demands were esthetical and functional. She complained her inability to do incision movement. She declined a TMJ pain at the moment of mastication.

- $\quad$ Full clinical exam was performed by orthodontists and maxillofacial surgeon.

- $\quad$ From the frontal view, she presented a parallelism at the horizontal lines of the face. The medial sagittal plan was deviated to the right side. She had atrophic nostrils, an underdevelopment cheekbones and a deep nasolabial fold. This was related to a retrusive maxilla eventually (Figure 1a).

- The profile was straight and orthofrontal with a slightly marked labiomental fold.

- $\quad$ She had a narrow and a shy smile. It was cuspid with a divated chin to the right (Figure $1 \mathrm{a}, \mathrm{b}$ ).

- The intraoral examination revealed a bilaterally molar and canine class III. A deviated lower incisor midline of $3 \mathrm{~mm}$ towards the right. Transversally, the patient showed a unilateral cross bite (on the right side) and an anterior crossbite. The mandibular arch had an oval form with crowded anterior teeth. In the other side, she had a lyre-shaped maxillary arch with crowding located at the premolar area. We observed also a huge tongue volume then the diagnosis of macroglossia was made (Figure 2: a, b, c, d, e).
- $\quad$ Cephalometrically, RB demonstrated a maxillary brachygnatism, a mandibular prognatism and a laterognatism. Then the anterior-posterior jaw relation was a severe class III skeletal with an ANB angle of $-1^{\circ}$ and an AoBo distance of $-10 \mathrm{~mm}$. Vertically, our patient had an hyperdivergent face with a GoGn angle of $49^{\circ}$. Although, an alveolar compensation was significant: a proclination of the the maxillary incisors with a retroclination of those mandibular.

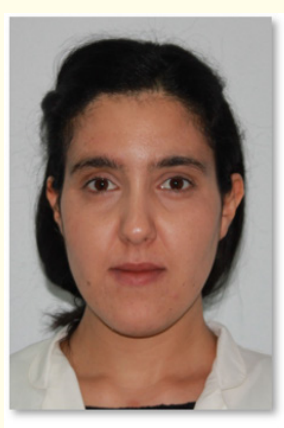

a) front view

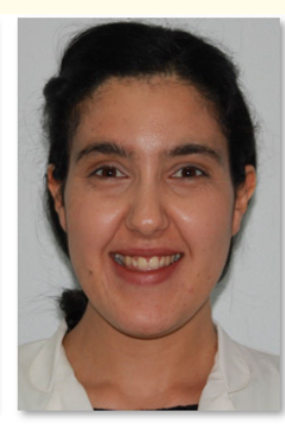

b) smile view

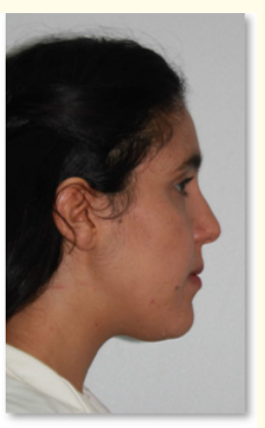

c) profil view
Figure 1: Before treatement (facial photographs).

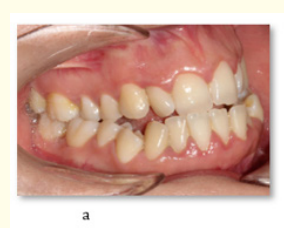

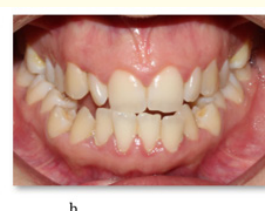

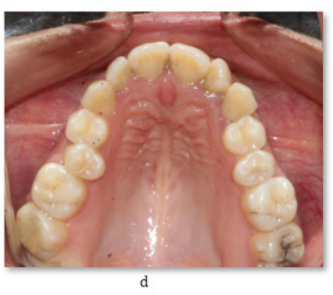

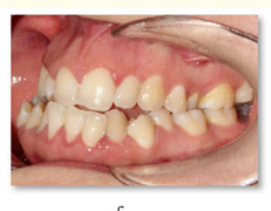

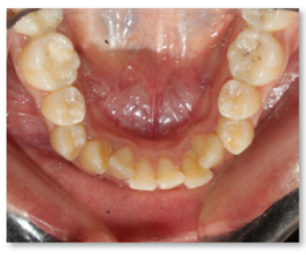

Figure 2: Pretreatment intraoral views.

\section{Treatment objectives}

- $\quad$ Procuring an expansion of the maxillary arch

- $\quad$ Solving the skeletal class III shift and acquiring a facial symmetry by doing a bimaxillary surgery 
- $\quad$ Treating the gummy smile running to an impaction surgery

- Resolving the dental crowding and the alveolar compensation

- Obtaining an occlusal adjustment, after obtaining gaining overjet and overbite using multi-bracket orthodontic appliances.

\section{Treatment options}

Because of the severe nature of her skeletal malocclusion, our treatment option was the following: a combined surgical orthodontic approach with a bimaxillary surgery: Lefort 1 osteotomy to advance and impact maxilla associated to an Obwegeser osteotomy to rotate mandibular jaw and obtain facial symmetry. This option needed to prosecute the typical presurgical orthodontic decompensation then was followed by conventional two-jaw surgery and completed with post-surgical finishing phase.

\section{Treatment progress}

- $\quad$ Succeeding acquiring informed consent, spacers were placed and the patient returned a week later for $0.022 \times 0.028$ bands for first and second molars and bonded brackets on the lower teeth.

- Initially, This first orthodontic phase (presurgical decompensation) began with levelling dental mandibular teeth using the following sequence of arches: 0.014", 0.016" then 0,018"NiTi. We have to add an interdental striping at the incisor zone which was indicated in presence of triangular incisors (Figure $3 \mathrm{a}, \mathrm{b}, \mathrm{c}, \mathrm{d}, \mathrm{e})$.

- Afterwards, with a stainless steel arch (0.018"), we did the maxillary expansion especially needed in the premolar area.

- Progressively, more rigid stainless steel arches were used to get ideal upper and lower arch coordination. That's why we utilized the following sequence of stainless steel arches: $0.017 \times 0.025 ", 0.018 \times 0.025 "$, and $0.019 \times 0.025$ " (Figure $4 \mathrm{a}, \mathrm{b}$, c, d, e).

- $\quad$ At that phase, a surgical simulation was needed. Therefore, alginate impressions were done and a semi adjustable articulator was used to perform simulation. Casts were mounted on the articulator. The maxilla was advanced by $5 \mathrm{~mm}$ and impacted by $3 \mathrm{~mm}$ anteriorly and $5 \mathrm{~mm}$ posteriorly. Mandibular cast was rotated by $3 \mathrm{~mm}$ to the left side. Two resin positioner were made: the first one refer to Lefort 1 osteotomy called intermediate positioner. Then a final positioner was constructed to get terminal position of the mandibular jaw (Figure 5).
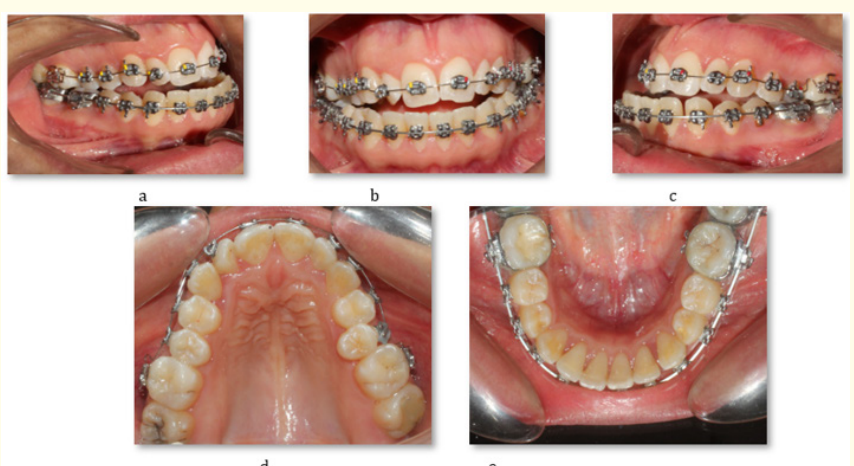

Figure 3: Intraoral views of the levelling phase (.014 stainless steel in the superior arch; .017x.025 NiTi in the inferior arch).

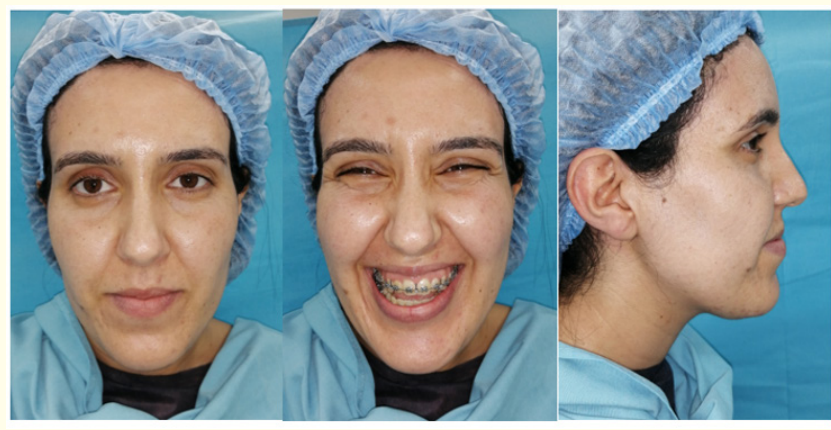

Figure 4: Presurgical facial photos.
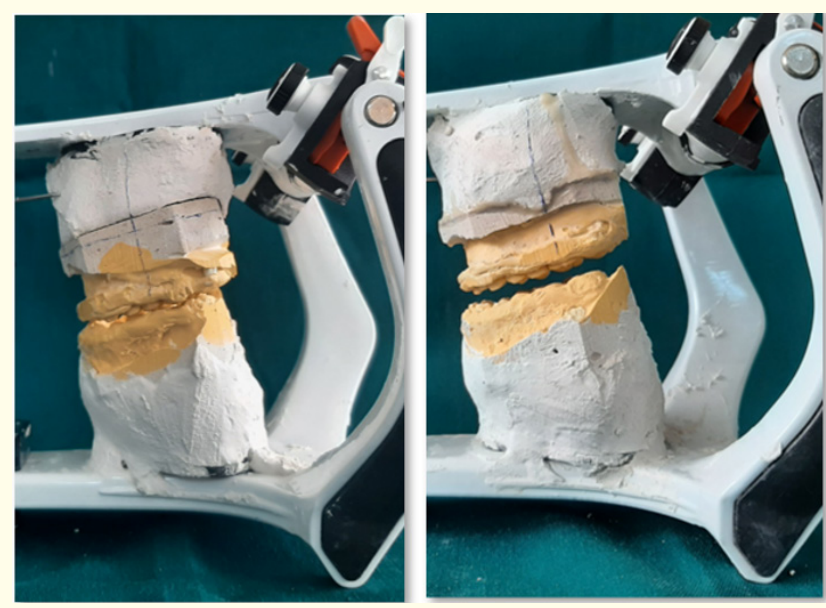

Figure 5: Surgery simulation. 
The surgical phase was assured by Professor R.M. That was preceded with many consultations and a long flow-up by both surgical team: maxillo-facial surgeon and Anesthesiologist Resuscitator to make sure the success of this intervention which lasted 5 hours (Figure 6).

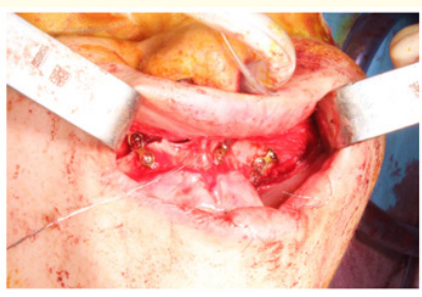

a) Lefort 1 osteotomy

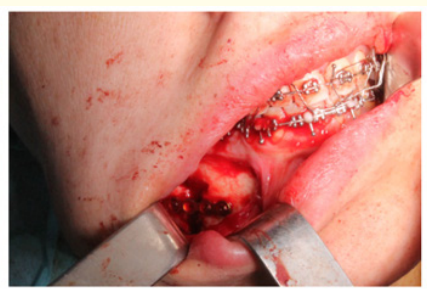

b) Obwegeser osteotomy
Figure 6: Double jaw surgery (a,b).

One month after recovery, we started finishing phase: closing posterior open bite. We applied intermaxillary elastics $(12 \mathrm{~mm} / 4 \mathrm{oz})$. To avoid pain, the elastics were applied only at night. Occlusal equilibration was also done to simulate dental abrasion surfaces and to aquire more occlusal stabilization. The finishing and the stabilisation phase was performed during 4 months to improve intercuspidation (Figure 7).

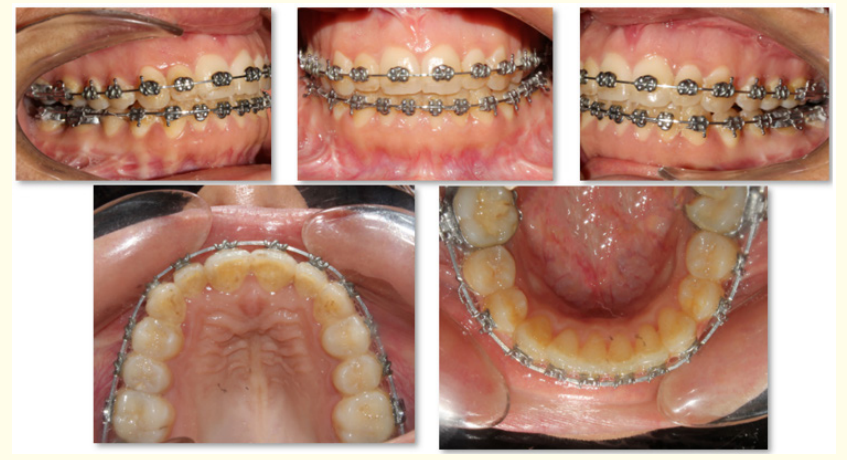

Figure 7: Intraoral views of the finishing phase.

After stabilization of the occlusal result, fixed retainers were bonded in superior and interior archs. Hawley retainer was added to ameliorate the biomechanics tongue during deglutition since the anatomic change after surgery.
Treatment results

Comparing the facial appearance before and after treatment, we can see from the front view how facial symmetry was obtained. We noticed a cheekbone prominence and a normal nasolabial fold. The profile was more harmonious with a correct nasolabial angle, normogenia and accurately gonial angle (Figure 8).
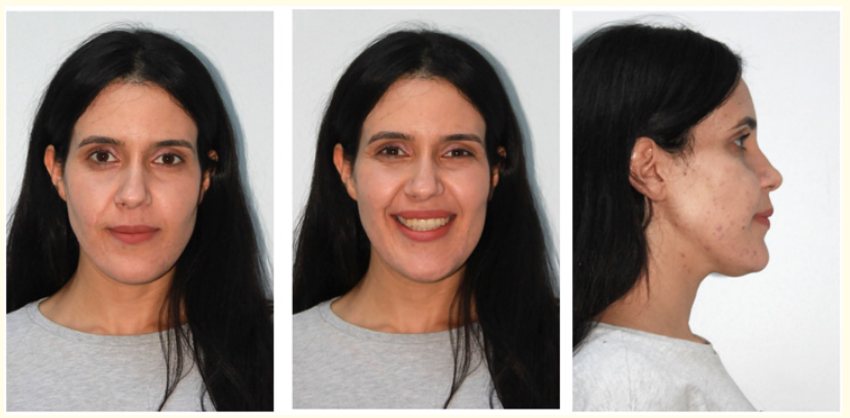

Figure 8: Final facial photographs.

Class I canine and molar was acquired with a positive relationship of the anterior tooth overlap (overjet $2.0 \mathrm{~mm}$, and overbite 2.0 $\mathrm{mm}$ ). Furthermore, horizontally, upper and lower midline were in accordance with facial median sagittal plane (Figure 9). Additionally, functional dynamic occlusion was procured. No TMJ disorder was detected. At last, a panoramic radiograph shows satisfying roots parallelism and good bone healing (Figure 10).

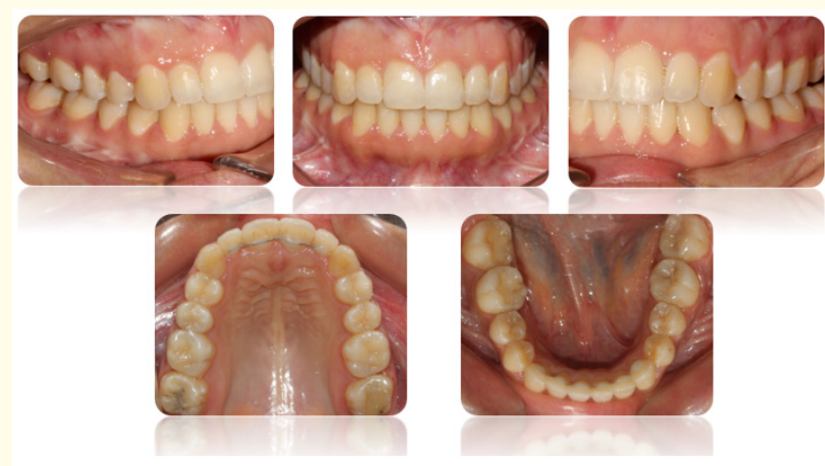

Figure 9: Final intraoral photographs. 

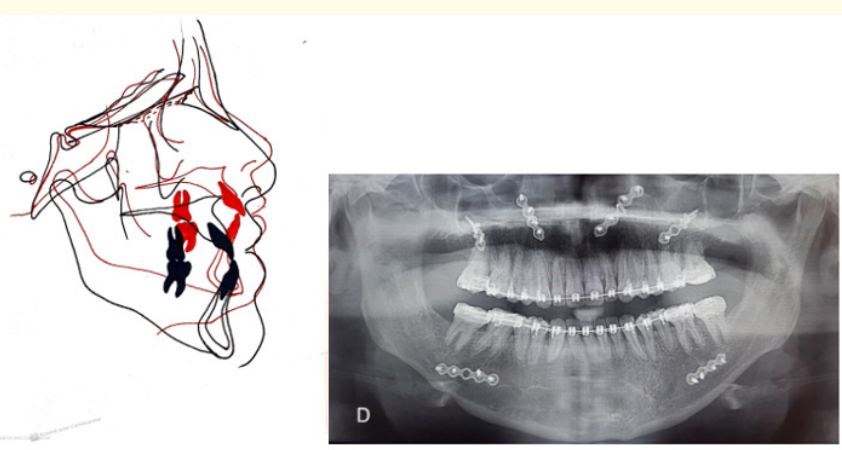

Figure 10: Panoramic radiograph and superposition schema.

\section{Discussion}

- Achieving planned treatment objectives was so much challenging. A multidisciplinary approach was needed in front of such severe skeletal malocclusion. Deep case discussions and good communication between orthodontist and maxillofacial surgeon are basics to succeed orthognatic treatments. Moreover, keeping in mind final result when decompensation phase was conducted; is crucial.

- The present case is a typical class III skeletal malocclusion owing to a deficient maxilla. That's why maxilla expansion was managed at first. In fact, RME is a common solution in such cases $[8,9]$. However, in our case intermaxillary suture was unable to be stimulated. Hence, expansion was ran using NiTi arches.

- In the other hand, according to many studies, the surgery first approach may be an interested treatment option [10]. Therefore, this approach allows shorter treatment duration [11] and a better psychosocial and esthetic features of class III skeletal malocclusion. Then, a better self-esteem for the patient is acquired. That option was not an appropriate approach in this case since we could not obtain a stable occlusion.

- Moreover, it was proven that Lefort one osteotomy advancement can provide a less constricted shape of pharyngeal airway [12]. According to Jinlong He., et al. bimaxillary surgery at treating class III skeletal malocclusion promotes less decrease on the upper airway than Obwegeser osteotomy setback [13].

- Indeed, according to Denadai., et al. the advancement of maxillary has an impact on 3D nasal morphometry. In fact, it was shown that the alar base width and nostril angle become larger after surgery. This was observed in our case and it is in favour of a better respiratory function [11,14].

\section{Conclusion}

In the present case report, the management of class III skeletal malocclusion owing to maxillary brachygnatia was shown. We first had to prepare and decompensate this class III in presurgical phase; in which a maxillary expansion was done. Double jaw surgery was conducted in order to correct the sagittal discrepancy and to achieve a facial symmetry. bimaxillary surgery should be the approach to choose in such cases to improve the volume of pharyngeal airway.

\section{Bibliography}

1. Salagnac JM. “Traitements des classes III". L'Orthodontie Française 77.2 (2006): 187-206.

2. Burns N R., et al. "Class III camouflage treatment: What are the limits?" American Journal of Orthodontics and Dentofacial Orthopedics 137.1 (2010): 9.e1-9.e13.

3. Francisco MARTINO., et al. "Surgical-orthodontic retreatment of a severe skeletal Class III malocclusion following an orthodontic camouflage". Dental Press Journal of Orthodontics 26.4 (2021).

4. Mariko Fuyamadaa., et al. "Reproducibility of maxillofacial landmark identification on three-dimensional cone-beam computed tomography images of patients with mandibular prognathism: Comparative study of a tentative method and traditional cephalometric analysis". The Angle Orthodontist 84.6 (2014): 966-973.

5. I Harakati., et al. "Frequency of malocclusions in 1383 cases of children supported by the National Health Insurance Fund". Revue Méditerranéenne d'Odonto-Stomatologie (R.M.O.S).2016.

6. Westwood P V., et al. "Long-term effects of Class III treatment with rapid maxillary expansion and facemask therapy followed by fixed appliances". American Journal of Orthodontics and Dentofacial Orthopedics 123.3 (2003): 306-320.

7. Toffol L D., et al. "Orthopedic Treatment Outcomes in Class III Malocclusion”. The Angle Orthodontist 78.3 (2008): 561-573.

8. Ngan P. "Early treatment of Class III malocclusion: Is it worth the burden?" American Journal of Orthodontics and Dentofacial Orthopedics 129.4 (2006): S82-S85. 
9. Tejashri Pradhan., et al. "Treatment Effect of Combined Surgical Maxillary Expansion and Mandibular Setback in Skeletal Class III". Contemporary Clinical Dentistry 12.2 (2021): 169173.

10. Barrabé A., et al. "Surgically assisted rapid palatal expansion in class III malocclusion: Our experience". Journal of Stomatology, Oral and Maxillofacial Surgery 186 (2018): 5.

11. Jinlong He., et al. "Impact on the upper airway space of different types of orthognathic surgery for the correction of skeletal class III malocclusion: A systematic review and meta-analysis". International Journal of Surgery 38 (2016): 31-40.

12. Yavuz Findık., et al. "Comparison of psychosocial and aesthetic features of class III malocclusion after orthognathic surgery: Conventional approach versus surgery-first approach". Journal of Stomatology, Oral and Maxillofacial Surgery (2021).

13. Giap HV., et al. "Pharyngeal Airway Morphology in Skeletal Class III With Mandibular Asymmetry is Improved After Bimaxillary Orthognathic Surgery". Journal of Oral and Maxillofacial Surgery 79.5 (2021): 1107-1121.

14. Rafael Denadai., et al. "Type of maxillary segment mobilization affects three-dimensional nasal morphology". Journal of Plastic Reconstructive and Aesthetic Surgery 74.3 (2020): 592-604.

\section{Assets from publication with us}

- Prompt Acknowledgement after receiving the article

- Thorough Double blinded peer review

- Rapid Publication

- Issue of Publication Certificate

- High visibility of your Published work

Website: www.actascientific.com/

Submit Article: www.actascientific.com/submission.php

Email us: editor@actascientific.com

Contact us: +919182824667

Citation: Chaima Attia., et al. "Skeletal Class III Treated with Double Jaw Surgery: A Case Report". Acta Scientific Dental Sciences 6.2 (2022): 25-30. 